\title{
Multifunctional load-bearing aerostructures with integrated space debris protection
}

\author{
Martin Schubert ${ }^{1,}{ }^{*}$, and Anthanasios Dafnis ${ }^{1}$ \\ ${ }^{1}$ Institute of Structural M echanics and Lightweight Design, RWTH A achen University, 52062 \\ A achen, Germany
}

\begin{abstract}
In the project multiSat multifunctional composite structures for satellite application have been developed. Functions such as protection against space debris, radiation shielding and passive thermal control have been integrated into the load-bearing composite spacecraft structure by use of suitable materials and components. Sandwich panels have been studied as representative structural parts of a conventional satellite structure. $M$ easures for increased space debris protection include the substitution of the conventional honeycomb core by 3D-printed aluminum cellular structures and the reinforcement of the sandwich panel by integration of high performance fabrics which effectively break up and catch impacting debris particles. This paper describes the development and design of multifunctional sandwich concepts with increased impact protection capability and presents the experimental results of hypervelocity impact testing with different types of CFRP sandwich panels.
\end{abstract}

\section{INTRODUCTION}

During development and production of conventional satellites usually structural, functional and protective functions are considered separately. The primary structure carries the mechanical loads and provides attachment points to the subsystems which are developed, tested, qualified and integrated into the satellite structure in a later separate step. On the one hand this distinct separation of functions increases the number of parts, total mass and installation volume, on the other hand it also drives up times and costs for production and launch of the satellite. In order to overcome this drawback the idea of a highly integrated multifunctional structure is pursued by integrating non-structural functions directly into the primary structure. The concept of a multifunctional structure allows for the reduction of the overall satellite mass and installation space required for subsystems. Additionally, it opens up new opportunities for highly integrative and standardized production processes and aims at lowering total costs and time for manufacturing, qualification and launch of a satellite.

In the project multiSat load-bearing lightweight structures for satellite application have been developed. In the framework of a comprehensive technological concept passive functions (protection against space debris, radiation shielding, passive thermal control) and active functions (energy and data transfer, reduction of micro-vibrations) have been

\footnotetext{
${ }^{*}$ Corresponding author: martin.schubert@ sla.rwth-aachen.de
} 
integrated by use of suitable materials, components and mechanisms. Due to their lightweight potential and multi-layer character composite materials are suitable for functional integration as each layer can be defined and designed to provide one or more specific functions. As Fig. 1 illustrates, the basic idea behind this concept is that different structural components are tailored and designed by use of specific materials and multilayer-sequences according to the individual structural and functional requirements.

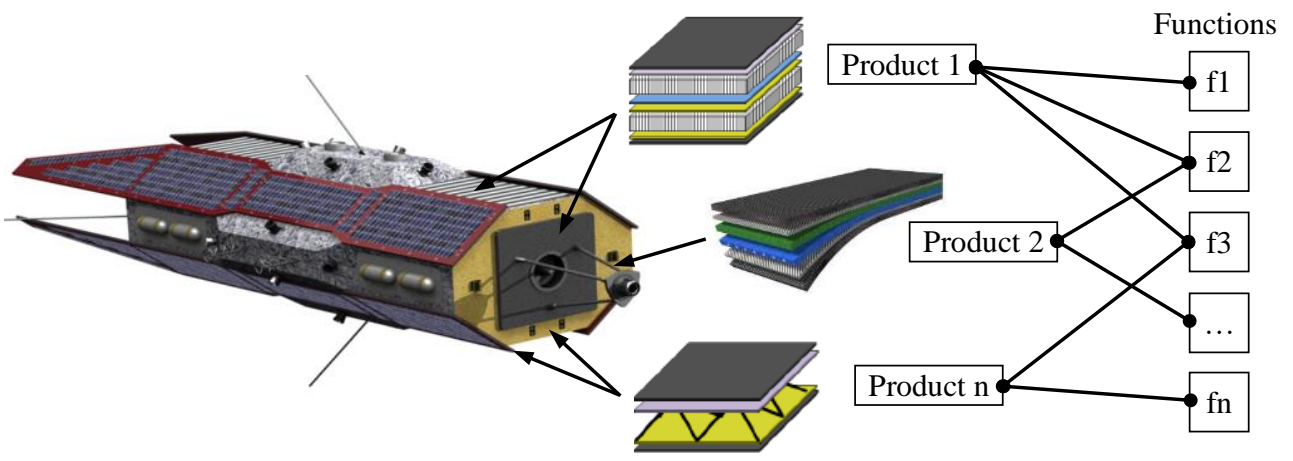

Fig. 1. Basic idea of a multifunctional composite satellite structure: individual multi-layer character of different structural components to meet specific structural and functional requirements.

Innovative manufacturing technologies such as additive manufacturing (AM) are increasingly playing an important role in the development of space structures. With 3D printing a part is built layer by layer according to a CAD model. Additively manufactured structures can have complex shapes which usually cannot be created with conventional manufacturing techniques. The freedom of design can be exploited to create structural parts which are optimized from both a structural-mechanical and multifunctional point of view because additional functions such as heat transfer or energy absorption can be embedded.

As a typical structural element of conventional satellite structures sandwich panels have been studied in this project to realize the functional integration. An overview about the technological concepts of the multifunctional structures with integrated passive and active functions, together with numerical simulations and experimental tests at coupon level is presented in [1]. The function focused on in the present paper is debris protection.

A major threat to space structures is the impact of space debris. In low earth average impact velocities of $7 \mathrm{~km} / \mathrm{s}$ and maximum impact velocities of up to $15 \mathrm{~km} / \mathrm{s}$ occur and even small debris particles with diameters of only few millimetres may cause detrimental damage to payloads and other subsystems of the satellite [2]. Only relatively big particles can be tracked to avoid collision by execution of evasion maneuvers. However, smaller particles are not detectable and have much higher fluxes. Furthermore, the amount of orbital debris is steadily increasing due to ongoing space activities. The growing risk of impact requires effective impact protection is for current and future satellite structures.

When considering the development of multifunctional structures two additional aspects are crucial: On the one hand, enhanced impact shielding is essential for protection of integrated components and mechanisms in order to maintain the multifunctionality of the structure during the whole mission period. On the other hand, the design for impact protection significantly influences the structural design of the primary structure. That is why debris protection is a design driver for the development of multifunctional satellite structures. In the following the development, analysis and testing of sandwich concepts with integrated space debris protection is presented. 


\section{SANDWICH CONCEPTS FOR IMPACT PROTECTION}

Space debris impact is a highly complex process which is characterised by generation, propagation and interference of shock waves within the target material and it can lead to incipient or detached spallation or even perforation of the rear wall of the shield [3]. Impact by space debris is usually categorized in two velocity regimes with a transition region in between them. Below an impact velocity of approximately $3-4.2 \mathrm{~km} / \mathrm{s}$ the impacting particle remains solid during the penetration of the shield whereas above approximately 7 $8.4 \mathrm{~km} / \mathrm{s}$ the temperatures and pressures upon contact become so high that the particle entirely vaporizes [4]. Monolithic shields are very inefficient debris shields because they require a relatively high wall thickness which results in significant weight penalty. The most effective protection against debris is achieved by double- or multi-wall shields with integrated flexible blankets made of high performance fabrics such as Nextel and Kevlar. These shields are typically used in manned spaceflight, i.e. the Whipple Shield or MultiShock Shield [3]. The outer layer, also called bumper, is relatively thin and causes break-up and vaporization of the impacting particle. A debris cloud is generated and it expands between the layers before impacting the rear wall, while distributing the residual kinetic energy onto a bigger surface, as is visualized in Fig. 2.
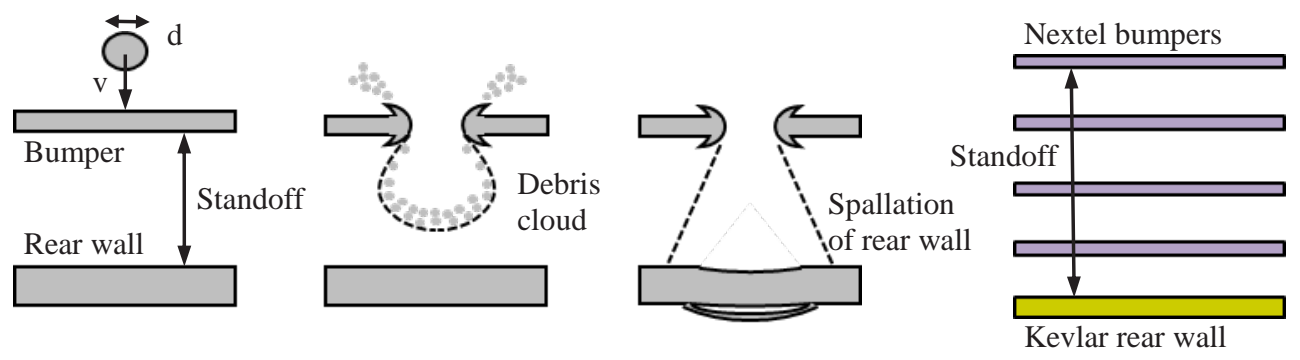

Fig. 2. Illustration of the hypervel ocity impact onto a double-wall shield (left); M ulti-Shock shield as example of a multi-wall debris shield (right).

The typical debris shields are separate, heavy and voluminous subsystems of the spacecraft and they are not part of the primary structure. A conventional sandwich structure inherently represents a double-wall shield. However, it usually provides significantly less protection compared to a typical Whipple shield because the honeycomb cell walls impede the expansion of the debris cloud which is called channelling effect [5]. The objective is to increase the debris protection capability of a composite sandwich as integral part of the satellite load-bearing structure without the addition of separate non-structural protective mechanisms. To achieve this suitable materials for increased protection against space debris shall be used and the core ideas of the multi-wall shield concepts used in manned spacecraft shall be applied to structural sandwich panels.

Typical material characteristics for enhanced debris shielding are one the one hand a superior particle fragmentation capability, and on the other hand a high energy absorption capability. Typically, Nextel, a ceramic fabric, is used in the bumper for effective break-up of a projectile whereas Kevlar or Dyneema, an aramid or polyethylene high strength fabric, are placed in the rear layers to slow down and catch the particles in the debris cloud [3]. Another effective option for increasing the impact resistance in the hypervelocity regime is the use of cellular sandwich core materials such as aluminium foam instead of honeycomb. The porous microstructure of the foam induces multiple collisions with the impactor leading to both repeated fragmentation and high energy absorption [5]. 
Based on the material selection and multi-wall concept two main approaches are considered to be most promising for improving the impact resistance of a sandwich panel: change of the sandwich core topology and addition of intermediate layers within the composite. Therefore, following measures are pursued for enhanced debris shielding:

- Consideration of the sandwich as effective double- or multi-wall shield

- Integration of Nextel and Kevlar fabrics as intermediate layers

- Replacement of honeycomb by additively manufactured aluminum cellular cores To assess the potential of the proposed measures for increased debris protection hypervelocity impact tests were carried out. For this purpose a selection of different and promising sandwich configurations, which are presented in Fig. 3, were produced taking into consideration the described measures. The reference configuration 1 is a conventional sandwich (areal density $0.37 \mathrm{~g} / \mathrm{cm}^{2}$ ) with CFRP face sheets (thickness $0.9 \mathrm{~mm}$ ) and aluminum honeycomb core (thickness $20 \mathrm{~mm}$ ). Configurations 1,2,3 have similar mass and compare the influence of the core topology on the impact behavior, whereas the configurations 4,5,6 are reinforced by additional intermediate layers and consequently heavier. All specimen have a quadratic shape of edge length $100 \mathrm{~mm}$ and the total core thickness is $20 \mathrm{~mm}$. The 3D printed aluminum cellular core structures were produced by selective laser melting (SLM).

1) Reference: conventional CFRP sandwich with aluminum honeycomb core

2) $3 \mathrm{D}$ printed aluminum pyramidal truss core, to allow for the free expansion of the debris cloud between the face sheets

3) $3 \mathrm{D}$ printed aluminum Kagome lattice core, to increase particle fragmentation and energy absorption, but at the same time allow for the expansion of the debris cloud

4) Same as configuration 2, but reinforced by Nextel in bumper layer to increase particle fragmentation, and Kevlar in rear wall for energy absorption

5) $3 \mathrm{D}$ printed lattice core as front core layer to induce particle fragmentation and expansion of debris cloud, and intermediate Kevlar layer for energy absorption

6) Double honeycomb core with intermediate layers: Nextel in bumper to increase particle fragmentation, and Kevlar in intermediate layer and rear wall for energy absorption. The tungsten layer (thickness $0.05 \mathrm{~mm}$ ) increases radiation shielding against electrons but it is comparably thin compared to all other layers.
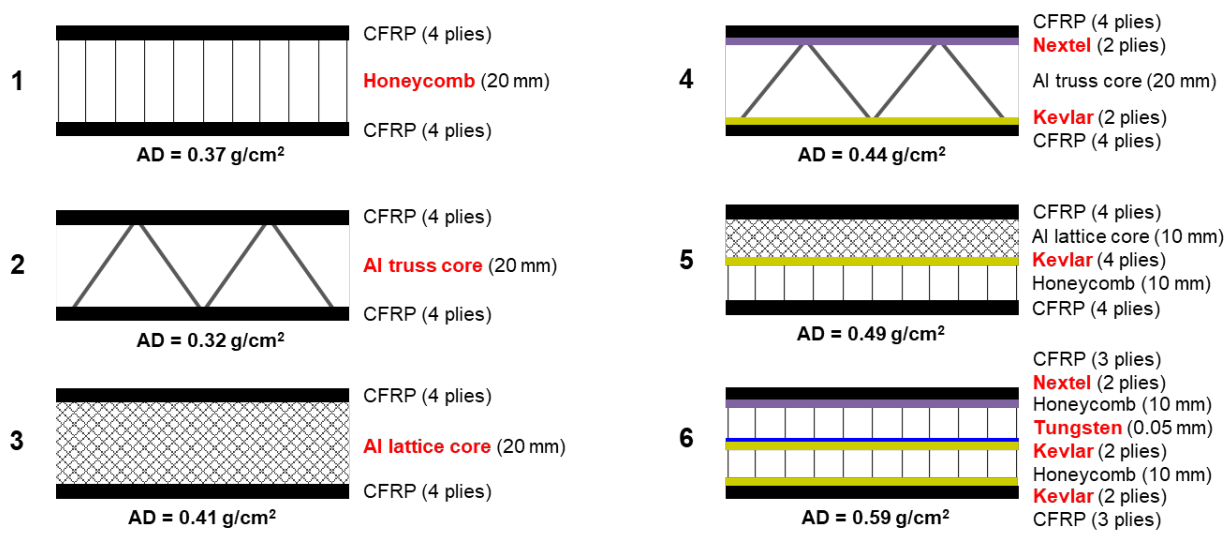

Fig. 3. Sandwich configurations for hypervel ocity impact testing: Increase of protection against space debris by variation of core topology $(1,2,3)$ and reinforcement by intermediate layers $(4,5,6)$. 


\section{HYPERVELOCITY IMPACT TESTING}

In order to study the impact behaviour of the sandwich configurations described in chapter 2 hypervelocity impact tests were conducted at the Fraunhofer Institute for High-Speed Dynamics, Ernst-Mach-Institut, EMI (Freiburg, Germany). For budget reasons an extensive, parametric and statistically verified test campaign was not feasible in the course of this project. However, even with the limited amount of specimen it was expected to demonstrate the potential of the tested sandwich configurations with regard to enhanced impact protection. The main objective was to study how the fragmentation and absorption of the impacting particle can be influenced and improved by use of alternative core topologies and additional intermediate layers.

A two-stage light gas was used for bombardment of the sandwich targets. This machine uses combusted gas as propellant and accelerates projectiles of a defined material and geometry up to speeds in the hypervelocity regime. The square sandwich targets of edge length of $100 \mathrm{~mm}$ are taped onto a so-called target holder which is placed inside the target chamber. The target holder consists of a front plate with a square hole which is connected via bolts to a so-called witness plate with a distance of $90 \mathrm{~mm}$ to the front plate. The black varnished witness plate catches the remaining particle fragments in case of penetration and allows for additional damage evaluation. Before impact the target chamber is evacuated to a pressure of 120 mbar to reduce atmospheric friction. Shortly after ignition a light barrier located at the end of the accelerator tube measures the velocity of the projectile and triggers a high-speed camera of type Shimadzu HPV-X which records the impact event. Table 1 lists the defined test parameters whereas Fig. 4 shows details of the test setup.

Table 1. Test specification for hypervelocity impact testing of the defined sandwich configurations.

\begin{tabular}{|l|l|l|}
\hline \multirow{4}{*}{ Projectile } & Geometry & Spherical, $\varnothing 1.25 \mathrm{~mm}$ \\
\cline { 2 - 3 } & Material & $\mathrm{A} 12072 \mathrm{~T} 4$ \\
\cline { 2 - 3 } & Impact velocity & $6.5-7.0 \mathrm{~km} / \mathrm{s}$ \\
\hline \multirow{2}{*}{ Recording } & Frames per shot & 128 \\
\cline { 2 - 3 } & Frame rate & $0.5-10 \mathrm{Mfps}$ \\
\hline
\end{tabular}
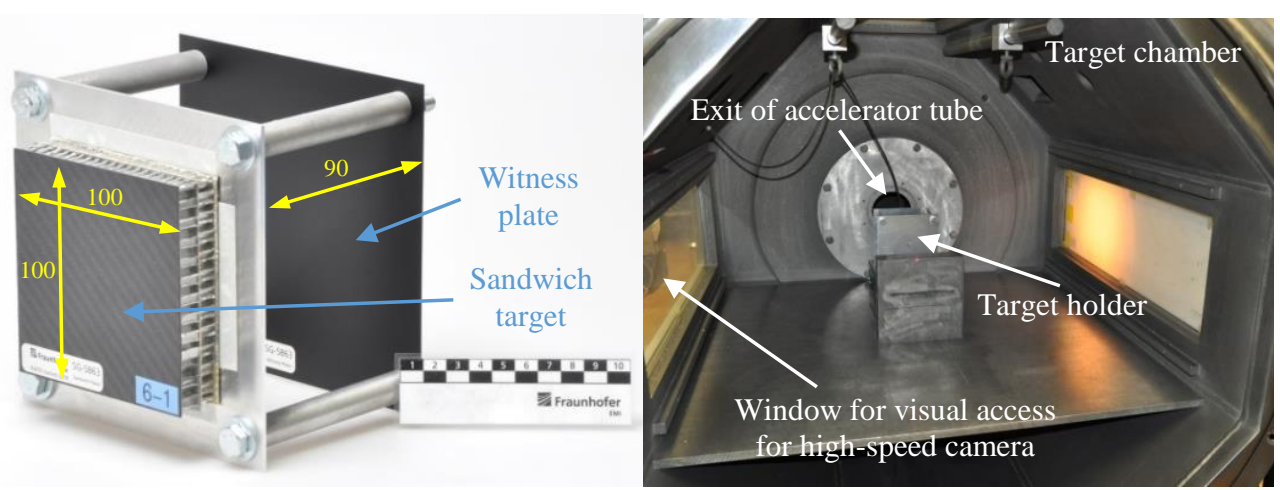

Fig. 4. Test setup for hypervelocity impact: Target holder with target (left), target chamber (right). 


\section{RESULTS OF IMPACT TESTS}

During the hypervelocity tests in total twelve shots on targets were performed which were suitable for studying and evaluating the impact behaviour of the different sandwich configurations. Even though on average only two shots per configuration were done new conclusions about the impact behaviour of these novel types of sandwich structures could be obtained. A satisfactory consistency of the results with regards to the impact event and the type and size of damage caused in the impacted target was observed, which provides important data and knowledge as empirical base for subsequent and more extensive hypervelocity test campaigns in the future. In the following the representative results for each of the six sandwich configurations are described and illustrated with help of Fig. 5.

Configuration 1: The conventional honeycomb sandwich plate shows the weakest impact protection performance as expected. A great portion of the impacting particle penetrates the sandwich structure in the form of a fragmented debris cloud. Additionally, a significant part of the CFRP rear wall is knocked out as secondary ejecta. The section cut at the location of impact reveals concentrated but severs damage of the honeycomb core which is indicative of the channelling effect caused by the honeycomb cell walls.

Configuration 2: The truss core allows for the almost unhindered formation of a debris cloud between the face sheets so that the particle fragments are distributed over a significantly larger area on the rear wall. However, the extent of damage is dependent of the location of impact as the nodes and struts of the truss core may interfere with the impacting particle. This is supported by the fact that for one shot minor penetration of the rear wall was observed after unhindered movement of the debris cloud through the core, whereas another shot showed less damage with no penetration at all after the particle and part of the debris cloud had hit a strut of the truss core.

Configuration 3: The lattice core both interacted with the debris cloud and allowed for its expansion. Similar to configuration 2 with truss core the diameter of the impacted area on the rear wall is much bigger than the diameter of the hole in the front face sheet. The lattice core for the most part has not suffered significant damage with exception of the area in vicinity of the impact hole. A similar minor penetration damage could be observed for the shot onto configuration 2 with no interference between the impactor and the truss core.

Configuration 4: The reinforced variant of configuration 2 shows neither penetration nor spallation of the rear wall. The inner surface of the rear face sheet indicates no structural damage as the surface of the Kevlar layer was only roughened and scratched by the impacting debris cloud which shows the superior energy absorption capability of the Kevlar fabric. The Nextel layer in the front face sheet is supposed to boost the vaporization of the impacting particle but from these results it is not possible to conclude whether and how much this layer has increased the fragmentation level of the debris cloud.

Configuration 5: No penetration of the rear wall occurred but the damage pattern differs for the two identical specimen. Within both targets the debris cloud interacted with the lattice core and expanded between the front face sheets and the intermediate Kevlar layer. However, in one case the intermediate Kevlar layer was penetrated and minor spallation on the rear face sheet was observed. In the other case the intermediate layer completely absorbed the debris cloud leaving the rear wall unimpacted but therefore causing deformation of the intermediate layer and the adjacent cell walls of the honeycomb core. Further study and evaluation of this discrepancy is necessary to identify the main influence parameters on the impact behaviour of such complex kind of structure. 
Configuration 6: The high-speed recording indicates a short-time and localized bulging of the rear face sheet at the projected location of impact, but neither penetration nor spallation of the rear wall occurred. This was in agreement with the expectations as this configuration is the heaviest and includes the most additional layers. Nevertheless, significant damage of the honeycomb core still is observed due to the channelling effect.
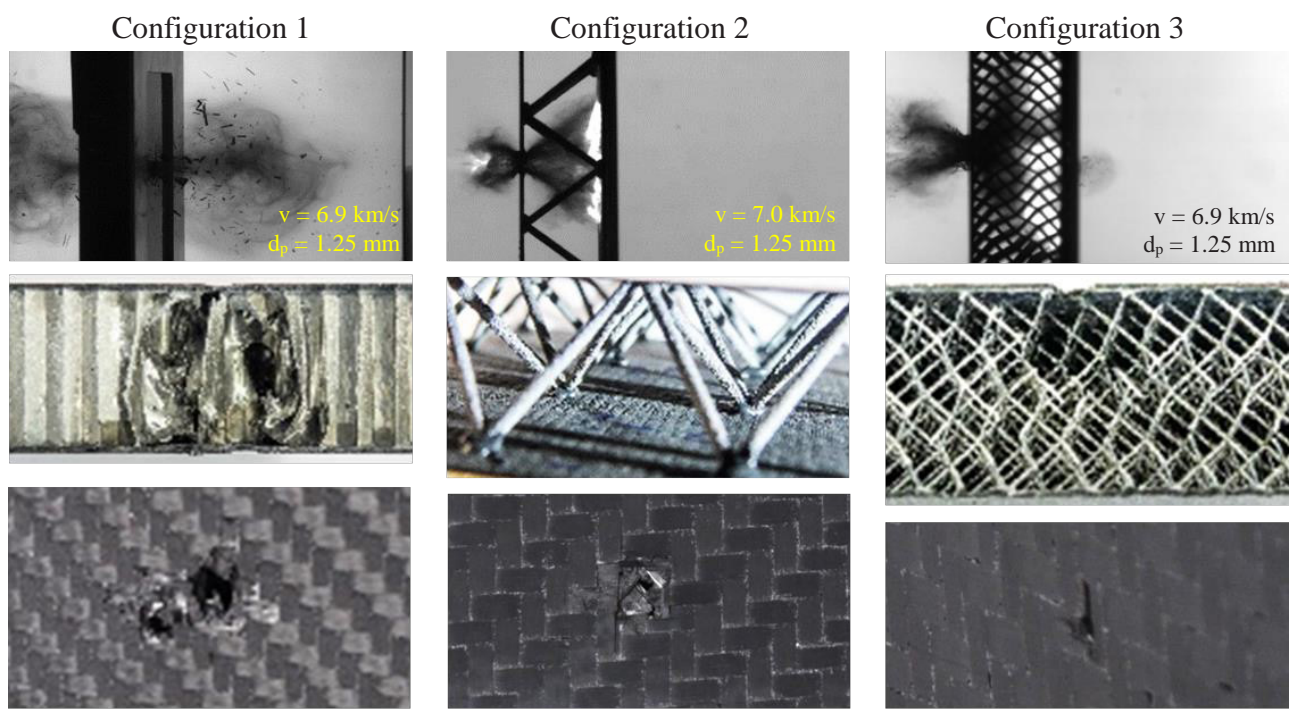

\section{Configuration 4}
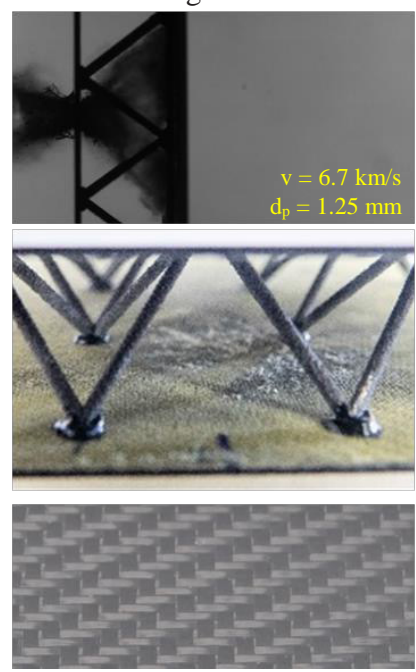

Configuration 5
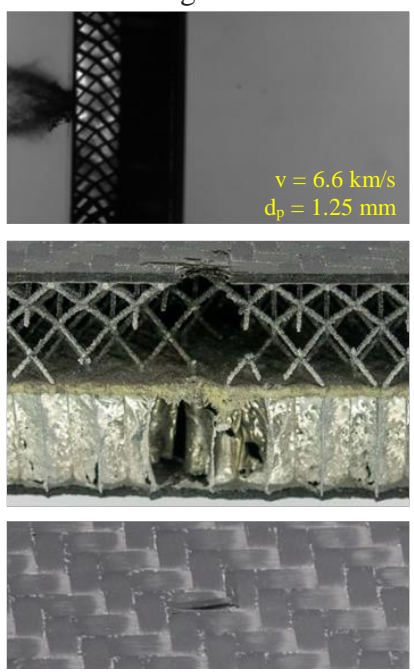

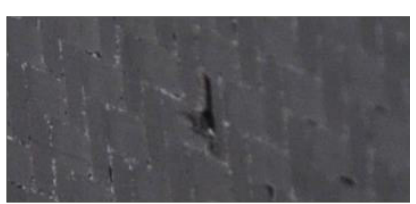

Configuration 6
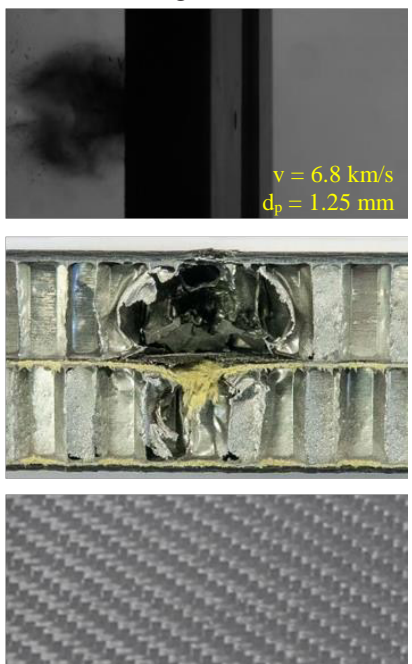

Fig. 5. Impact behaviour for one representative specimen of each sandwich configuration: side view during impact (top); section cut at location of impact (center); damage on the rear face sheet (bottom).

Even though the results are not based on statistical evidence due to the limited amount of tested specimen, significant differences in the impact behaviour and shielding performance were overserved which allow to evaluate the potential of the tested sandwich configurations for increased impact protection. The main findings are: 
- The core topology significantly influences the impact behaviour and the damage pattern in a CFRP sandwich panel. By replacing the conventional aluminum honeycomb core with $3 \mathrm{D}$ printed aluminium cellular cores the debris shielding performance of the sandwich can be increased without weight penalty.

- It is not possible to conclude whether a truss- or lattice-type cellular structure is favourable as core material concerning impact protection. Both allow for the expansion of the debris cloud which is crucial for shielding against space debris.

- Additional intermediate layers increase the impact protection capability of a composite sandwich panel, but with weight penalty. However, they do not prevent channelling of the debris cloud if a honeycomb core is used.

- Kevlar shows a high energy absorption capability and is suitable for use as intermediate layer or part of the rear wall in a load-bearing CFRP sandwich panel.

\section{CONCLUSION AND OUTLOOK}

In the course of the project multiSat composite sandwich structures with integrated debris protection have been developed and experimentally studied by conducting hypervelocity impact tests on different sandwich panel configurations. Feasibility was shown, that basic concepts for weight efficient debris shields used in manned spacecraft can be applied and integrated into load-bearing CFRP sandwich panels for satellite application. Materials and structural design concepts for improving the impact behaviour of conventional composite sandwich panels were identified. By changing the core typology by use of additively manufactured cellular aluminium structures and integrating intermediate layers of high performance fabrics the formation, expansion and absorption of the debris cloud can be influenced in order to increase shielding performance in case of space debris impact.

This project could only provide a limited amount of hypervelocity tests. However, high potential of novel sandwich designs for enhanced debris shielding for future satellites was demonstrated. Detailed identification and evaluation of the impact and damage parameters relevant for systematic impact damage assessment is currently on-going. Plans for an extensive and systematic hypervelocity impact test campaign are being pursued and aim at continuing research within the framework of a national project in collaboration with ESA.

The project multiSat was a German national project promoted by the DLR Space Administration and the Federal Ministry of Economic Affairs and Energy. Funding by these entities has been granted in the framework of the INNOspace ${ }^{\circledR}$ Masters 2016 initiative under the slogan "Satellite 4.0". The duration of the project was two years from October 2016 to September 2018.

\section{References}

1. M. Schubert, S. Perfetto, A. Dafnis, D. M ayer, H. Atzrodt, Development and design of multifunctional lightweight structures for satellite applications, Proceedings of the 69th International Astronautical Congress (2018)

2. W. Ley, K. W ittmann, W. Hallmann (Ed.), Handbook of Space Technology, 1 (2009)

3. E.L. Christiansen, S. Ryan, Handbook for Designing M M OD Protection, NASA/TM 2009-214785 (2009)

4. S. Ryan, E.L. Christiansen, Micrometeoroid and Orbital Debris (MMOD) Shield B allistic Limit A nalysis Program, NA SA/T M -2009-214789 (2010)

5. S. Ryan, E.L. Christiansen, Hypervelocity Impact Testing of Aluminum Foam Core Sandwich Panels, N A SA/TM -2015-218593 (2015) 